\title{
Deep neural network-based surrogates for Surface Complexation Models of oxide/electrolyte interfaces
}

\author{
CHUNHUI LI ${ }^{1}$, STEVEN FARRELL ${ }^{1}$, BENJAMIN \\ GILBERT $^{2}$ AND PIOTR ZARZYCKI ${ }^{1}$ \\ ${ }^{1}$ Lawrence Berkeley National Laboratory \\ ${ }^{2}$ Lawrence Berkeley National Lab \\ Presenting Author: chunhuili@lbl.gov
}

Oxide/electrolyte interfaces are the most common and largest interfaces on Earth. When oxide particles are immersed in an aqueous solution, the interfacial charge distribution, known as the electric double layer (EDL), develops. These interfaces are typically probed using electrophoretic, potentiometric, and electrochemical methods. Fits to these results using Surface Complexation Modeling (SCM) can provide insight into surface binding equilibrium constants, and spatial charge distribution at the interface.

However, the parameterization procedure of fitting SCM to experimental data is time-consuming and it remains a challenge to discover unique and transferable SCM parameters to describe the EDL. We hypothesized that deep neural networks (DNN) could greatly improve the information obtained from fits to experimental data that probe EDL structure. Specifically, training a DNN on data from all known SCM data could permit fits to any single dataset to be more consistent and transferable across all mineral-water interfaces. To test this approach, we calculated a large number of synthetic SCM data using the $2 \mathrm{pK}$-Triple Layer Model, using parameters for many common mineral surfaces, and used these synthetic data to train a surrogate DNN. By doing so, the SCM-trained DNN is capable of ultra-fast refinement of SCM parameters, enabling the unique solution of model parametrization from test experimental or synthetic data. The SCM-DNN is capable not only of finding the global minimum in a large parameter-space but also restricts the optimization domains to only physically and chemically sound subregions.

We demonstrate that a DNN-based SCM for oxide/electrolyte interfaces is feasible and cost-effective in comparison to conventional fitting paradigms. This DNN-SCM is an example of a general class of surrogates that could replace existing models used to analyze and interpret experimental observations. Such models can substantially reduce the computational cost, hardware demands and accelerate data analysis. 